\title{
ANALYSIS OF DIFFUSION-WEIGHTED IMAGING (DWI) PARAMETERS AND ADC MAP FOR BREAST INJURY STUDY
}

Ilse Franco de Oliveira¹, Camila Leal Diniz¹, Rosemar Macedo Sousa, Cristina Pinto Naldi Ruiz², Paulinelly Messias de Almeida², Ruffo de Freitas-Junior ${ }^{1}$

'Universidade Federal de Goiás - Goiânia (GO), Brazil.

${ }^{2}$ Clínica São Marcelo - Goiânia (GO), Brazil.

Objective: To optimize the parameters of effectiveness of the use of the diffusion sequence in magnetic resonance imaging examinations of the breast. Methodology: Prospective study of qualitative analysis of the DWI sequence, performed with three volunteers $(\mathrm{A}, \mathrm{B}$, and $\mathrm{C}$ ) in $1.5 \mathrm{~T}$ magnetic resonance equipment (X, Y, and Z), with bilateral synergy coil containing four channels each, dedicated to the study of the breasts for further analysis of the signal intensity parameters of the broadcasting sequence. Results: In the B equipment Y test, there was a cut of the nipple region of the left breast, requiring adaptation of the FOV for homogeneity of the field, allowing for better image quality and reduction of artifacts. The images from test $\mathrm{A}$ equipment $\mathrm{X}$ showed loss of signal, requiring adjustments to the matrix resolution, with a corresponding reduction in the echo time (TE) and an increase in the number of acquisitions (NEX) to optimize the signal-tonoise ratio (SNR). The choice of four-channel synergy coils in the three tests favored the greater intensity and uniformity of the magnetic field, and due to the fact that it is dedicated to the breasts bilaterally, it was possible to acquire simultaneous images, reducing the examination time. In the images acquired in the axial plane, changes were made to the phase coding, defined from left to right, to decrease cardiac and respiratory movements. Conclusion: After analyzing the DWI images and their respective ADC maps, there was a need to build a Decision Matrix to guide the standardization of $b$ values to avoid the influence of the perfusion phenomenon and consequently a false representation of the tissues in the ADC map.

Keywords: Breasts; Diffusion; Magnetic Resonance. 